\title{
Prevalence of high blood pressure in Iranian adults based on the 2017 ACC/AHA guideline
}

\author{
Mostafa Hosseini ${ }^{1}$, Mehdi Yaseri ${ }^{1}$, Hadi Asady ${ }^{2,3}$, Ahmed Eleojo Musa ${ }^{4}$, Jalil Koohpayehzadeh ${ }^{5}$, \\ Ali Rafei ${ }^{6}, K$ oorosh Etemad ${ }^{6,7}$, Mohammad Mehdi Gouya ${ }^{6}$, Fereshteh Asgari ${ }^{6}$, Mahmoud Yousefifard*8
}

\section{Abstract}

Background: : In 2017, American College of Cardiology (ACC) and the American Heart Association (AHA) presented a new guideline for assessing blood pressure in adults. This study aimed to assess the prevalence of hypertension in Iranian adults based on ACC/AHA 2017 guideline.

Methods: Data from 9801 Iranian adults (59.2\% women) aged between 20-69 years were obtained from the sixth round of National Surveillance of Risk Factors of Non-Communicable Diseases (SuRFNCD) performed in 2011. Blood pressure was classified as normal, elevated blood pressure, and stage 1 and 2 hypertension using a weighted analysis and 2017 ACC/AHA guidelines. Data were presented as prevalence and $95 \%$ confidence interval $(95 \% \mathrm{CI})$. All analyses were performed in Stata/SE 14.0.

Results: Overall prevalence of hypertension in Iranian men was 52.0\%. Also, 32.9\% (95\% CI: 29.9-36.0) and 19.1\% (95\% CI: 16.9 21.6) of men had stage 1 and 2 hypertension, respectively. In addition, $44.3 \%$ of women had hypertension, of whom $26.3 \%$ (95\% CI: 24.5 - 28.2) had stage 1 and 18.0\% (95\% CI: 16.1-20.1) stage 2 hypertension. Furthermore, $16.5 \%$ (95\% CI: 14.4-18.9) and 9.6\% (95\% CI: 7.86-11.7) of men and women had elevated blood pressure, respectively.

Conclusion: The findings of this study indicated that adopting the 2017 ACC/AHA guidelines showed a higher prevalence of adult hypertension $(48.2 \%)$ in Iran. In this study, the prevalence of hypertension in men was higher than in women, which was steadily increased by age in older adults in both sexes.

Keywords: Adult hypertension, ACC/AHA guidelines, Prevalence, Iran, High blood pressure

Conflicts of Interest: None declared

Funding: Tehran University of Medical Sciences

*This work has been published under CC BY-NC-SA 1.0 license.

Copyright $\odot$ Iran University of Medical Sciences

Cite this article as: Hosseini M, Yaseri M, Asady H, Eleojo Musa A, Koohpayehzadeh J, Rafei A, Etemad K, Gouya MM, Asgari F, Yousefifard M. Prevalence of high blood pressure in Iranian adults based on the 2017 ACC/AHA guideline. Med J Islam Repub Iran. 2019 (2 Apr);33:26. https://doi.org/10.47176/mjiri.33.26

\section{Introduction}

Hypertension is one of the major health concerns in developing countries, especially among the adult population (1-3). It has been predicted that by the year $2025,75 \%$ of hypertensive patients will be from developing countries

Corresponding author: Dr Mahmoud Yousefifard, yousefifard.m@iums.ac.ir

1. Department of Epidemiology and Biostatistics, School of Public Health, Tehran University of Medical Sciences, Tehran, Iran

2. Department of Occupational Health Engineering, School of Health, Isfahan University of Medical Sciences, Isfahan, Iran

3. Social Determinants of Health Research Center, Saveh University of Medical Sciences, Saveh, Iran

4. Department of Medical Physics and Biomedical Engineering, Tehran University of Medical Sciences, International Campus, Tehran, Iran

5. Department of Community Medicine, Iran University of Medical Sciences, Tehran, Iran

6. Center for Disease Control, Ministry of Health and Medical Education, Tehran, Iran

7. Department of Epidemiology, School of Public Health, Shahid Beheshti University of Medical Sciences, Tehran, Iran

8. Physiology Research Center, Faculty of Medicine, Iran University of Medical Sciences, Tehran, Iran
(4). In 2017, a new guideline for prevention, detection, evaluation, and management of high blood pressure in adults was published by the American College of Cardiology and the American Heart Association (ACC/AHA) (5).

$\uparrow$ What is "already known" in this topic:

In 2017, American College of Cardiology (ACC) and the American Heart Association (AHA) presented a new guideline for assessment of blood pressure in adults. However, the prevalence of hypertension in Iranian population is unclear according to the ACC/AHA 2017 guideline.

$\rightarrow$ What this article adds:

The findings of this study indicated that adopting the 2017 ACC/AHA guidelines showed a higher prevalence of adult hypertension (48.2\%) in Iran. Prevalence of hypertension in men was higher than in women. This prevalence was steadily increased by age in older adults in both sexes. 
In their guideline, blood pressure (BP) was classified in 4 categories of nor mal, elevated, and stage 1 and 2 hypertension.

In the new definition of ACC/AHA, the threshold for the definition of elevated blood pressure and hypertension is $10 \mathrm{mmHg}$ lower than the definition of the Joint National Committee for Prevention, Detection, Evaluation and Treatment of High Blood Pressure (JNC 7, 2004) guideline (6). This new classification can identify people who are prone to hypertension at an early stage of the disease, which reduces the burden of disease and improves the effectiveness of preventive measures.

As a result of these new guidelines, there have been obvious changes in the prevalence of hypertension among adults around the globe. For instance, in a study by Muntner et al, the proportion of adults in the US with high BP increased from $31.9 \%$ to $45.6 \%$ (7). In another study, Khera et al found an increase in the prevalence of hypertension by $26.8 \%$ in the US and $45.1 \%$ in China in adults aged 45 to 75 years (8).

Iran is a developing middle Eastern country with a population of over 80 million, with diverse cultures, socioeconomic status, and lifestyle, which are all factors that affect hypertension (9). According to a 2011 survey, 25.8\% and $27.6 \%$ of Iranian men and women had hypertension, respectively (2). For most Iranian adults, diet is a major risk factor followed by high BP and overweight (10). Hence, the aim of this study was to assess the impact of diet on the prevalence of hypertension in Iranian adults using the new ACC/AHA guidelines. This will go a long way to better guide future health policy interventions in managing hypertension.

\section{Methods \\ Study design and participants}

In this cross sectional study, data from 9801 Iranian adults aged 20-69 years were obtained from the sixth round of National Surveillance of Risk Factors of NonCommunicable Diseases (SuRFNCD) performed in 2011 by the Iranian Center for Disease Control and Prevention (11). Institutionalized people (such as soldiers and hospitalized patients) and nomadic tribes were excluded from the study. Data were obtained through a nationwide survey across all 31 provinces of non-hospitalized and noninstitutionalized population of Iran. Briefly, a 4-stagesampling scheme was used to collect data on 6- to 70year-old Iranians. Provinces were taken as primary sampling units (first stage) and villages or cities as secondary sampling units (second stage) in this survey. Then, random 10-digit postal codes were selected from each secondary sampling unit (third stage). Finally, the participants were selected using KISH method (fourth stage).
Ethic Committee of Tehran University of Medical Sciences approved the protocol of the present study. In this study, the researchers adhered to Helsinki declaration.

\section{Measurement and classification of blood pressure}

In National Surveillance of Risk Factors of NonCommunicable diseases, blood pressure measurements were done twice with 1-minute interval, and their average values were used to reduce bias. Mercury sphygmomanometer was used to measure BP after a relaxation period of 5 minutes. A $40 \%$ arm length covering $80 \%$ to $100 \%$ of the full arm was used as the bladder width. Afterwards, the cuff was deflated by positioning the stethoscope on the brachial artery as well as increasing the bladder pressure by $30 \mathrm{mmHg}$. Systolic blood pressure (SBP) was determined by the first Korotkoff (K1) sound, while the fifth represented the diastolic blood pressure (DBP) (1).

From the SuRFNCD 2011 dataset, information on demographic characteristics (age and sex) and BP measurements of those aged 20-69 years was extracted to classify participants as having either normal, elevated, or stage 1 or 2 hypertension. These classifications were based on the 2017 ACC/AHA guidelines on prevention, diagnosis, evaluation, and management of hypertension in adults (Table 1).

\section{Statistical analysis}

The number and percentage of individuals were computed according to gender and age groups. Because of the sampling scheme, weighted analysis was employed and the individual's sampling weights were computed by multiplying the selection probabilities in each step of the survey. The prevalence of blood pressure categories and its associated $95 \%$ confidence intervals (95\% CIs) within the age groups were estimated for men and women using complex sample survey analysis. All analyses were performed in Stata/SE 14.0 (StataCorp, College Station, TX, USA).

\section{Results}

In this study, data on 9801 participants, 3999 (40.8\%) men and $5802(59.2 \%)$ women, were assessed. Participants' age ranged from 20 to 69 years. The number (percentage) of study population according to age groups and sex is given in Table 2 .

Using the ACC/AHA categories, the prevalence of stage 1 and 2 hypertension was found to be $29.6 \%$ and $18.6 \%$, respectively, in Iranian adults (The overall prevalence of hypertension was $48.2 \%$ ). Furthermore, $13.1 \%$ of the participants had elevated hypertension while only $38.7 \%$ had normal blood pressure.

Table 3 shows the prevalence of hypertension in Iranian

Table 1. New blood pressure categories in adults according to ACC/AHA guidelines

\begin{tabular}{lc}
\hline Category & Definition \\
\hline Normal & SBP $<120 \mathrm{mmHg}$ and DBP $<80 \mathrm{mmHg}$ \\
Elevated & SBP between 120-129 mmHg and DBP $<80 \mathrm{mmHg}$ \\
Hypertension & \\
$-\quad$ Stage 1 & SBP between 130-139 mmHg or DBP between $80-89 \mathrm{mmHg}$ \\
$-\quad$ Stage 2 & SBP $\geq 140 \mathrm{mmHg}$ or DBP $\geq 90 \mathrm{mmHg}$ \\
\hline SBP: Systolic Blood Pressure; DBP: Diastolic Blood Pressure &
\end{tabular}




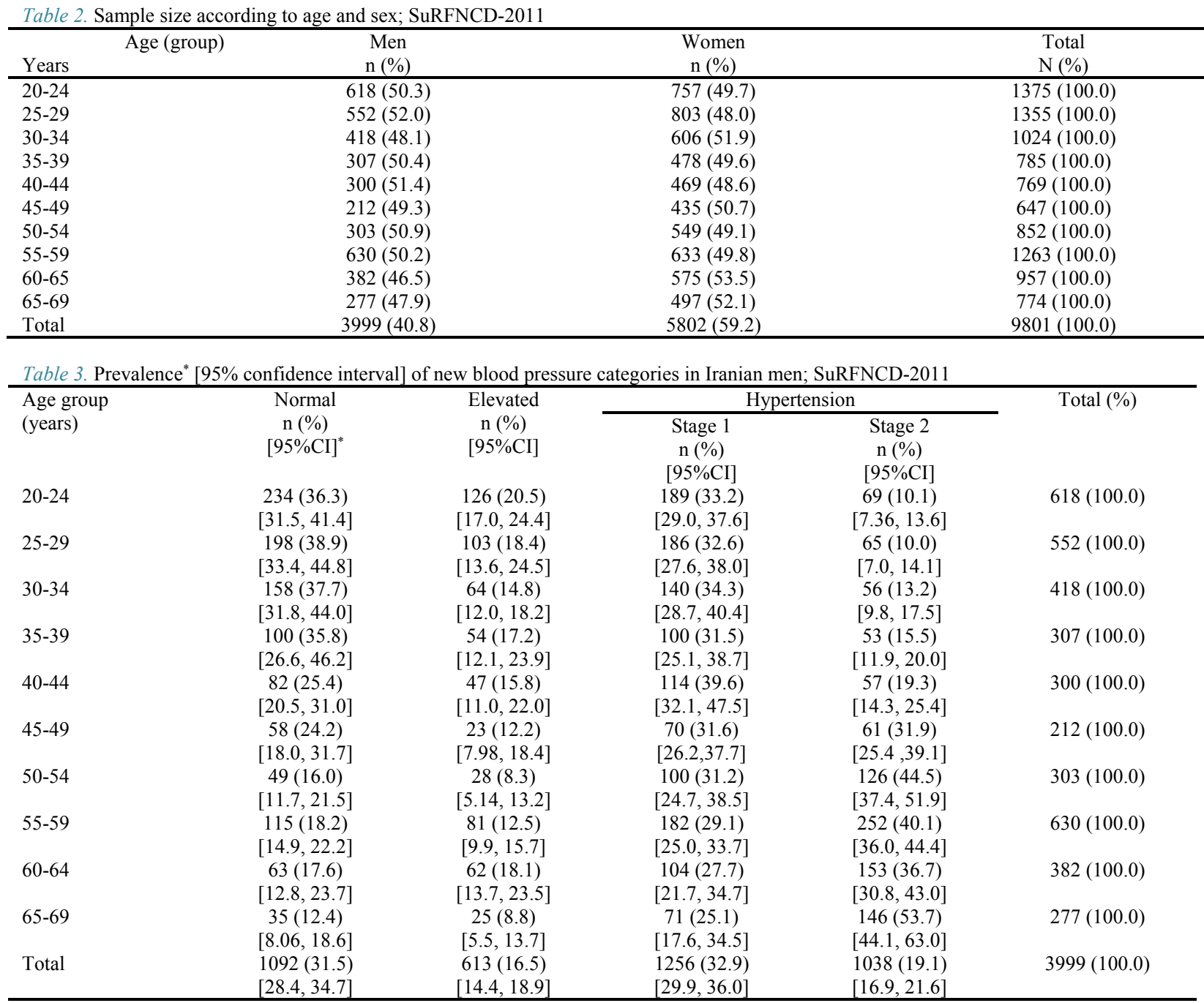

*Weighted analysis; CI: Confidence interval.

men according to age groups. Based on the results, 52.0\% of the male population were estimated to be hypertensive. Of them, 32.9\% (95\% CI: 29.9-36.0) and 19.1\% (95\% CI: 16.9-21.6) had stage 1 and 2 hypertension, respectively. The prevalence of stage 2 hypertension was higher in older men (45 years and older) compared to the younger age groups. However, the prevalence of stage 1 hypertension was not significantly different across age groups.
Table 4 shows the prevalence of hypertension in Iranian women according to age groups. Overall, about $44.3 \%$ of the female population had hypertension. Of them, $26.3 \%$ (95\% CI: 24.5-28.2) had stage 1 and 18.0\% (95\% CI: 16.1-20.1) stage 2 hypertension. Similar to the men, the prevalence of stage 2 hypertension also increased in women with age. Moreover, the prevalence of stage 1 hypertension was close across age groups except for 20-24 age

\begin{tabular}{|c|c|c|c|c|c|}
\hline \multirow{2}{*}{$\begin{array}{l}\text { Age group } \\
\text { (years) }\end{array}$} & \multirow{2}{*}{$\begin{array}{c}\text { Normal } \\
\mathrm{n}(\%) \\
{[95 \% \mathrm{CI}]^{*}}\end{array}$} & \multirow{2}{*}{$\begin{array}{c}\text { Elevated } \\
n(\%) \\
{[95 \% \mathrm{CI}]}\end{array}$} & \multicolumn{2}{|c|}{ Hypertension } & \multirow[t]{2}{*}{ Total (\%) } \\
\hline & & & $\begin{array}{c}\text { Stage } 1 \\
n(\%) \\
{[95 \% \mathrm{CI}]}\end{array}$ & $\begin{array}{c}\text { Stage } 2 \\
\mathrm{n}(\%) \\
{[95 \% \mathrm{CI}]}\end{array}$ & \\
\hline $20-24$ & $\begin{array}{c}513(68.4) \\
{[63.4,73.1]}\end{array}$ & $\begin{array}{c}72(10.0) \\
{[7.31,13.7]}\end{array}$ & $\begin{array}{c}146(18.3) \\
{[15.1,22.0]}\end{array}$ & $\begin{array}{c}26(3.3) \\
{[2.08,5.07]}\end{array}$ & $757(100.0)$ \\
\hline $25-29$ & $\begin{array}{c}464(56.5) \\
{[51.7,61.3]}\end{array}$ & $\begin{array}{c}61(8.2) \\
{[5.83,11.3]}\end{array}$ & $\begin{array}{c}220(27.7) \\
{[23.3,32.7]}\end{array}$ & $\begin{array}{c}58(7.6) \\
{[5.9,9.7]}\end{array}$ & 803 (100.0) \\
\hline $30-34$ & $\begin{array}{c}304(47.8) \\
{[42.2,53.5]}\end{array}$ & $\begin{array}{c}58(9.2) \\
{[6.36,13.3]}\end{array}$ & $\begin{array}{c}166(30.5) \\
{[25.2,36.4]}\end{array}$ & $\begin{array}{c}78(12.4) \\
{[9.6,15.9]}\end{array}$ & $606(100.0)$ \\
\hline $35-39$ & $\begin{array}{c}207(44.1) \\
{[38.2,50.1]}\end{array}$ & $\begin{array}{c}44(8.2) \\
{[5.54,12.1]}\end{array}$ & $\begin{array}{c}137(28.6) \\
{[24.8,32.7]}\end{array}$ & $\begin{array}{c}90(19.1) \\
{[14.9,24.1]}\end{array}$ & $478(100.0)$ \\
\hline $40-44$ & $\begin{array}{c}153(33.7) \\
{[28.3,39.5]}\end{array}$ & $\begin{array}{c}57(11.4) \\
{[8.24,15.7]}\end{array}$ & $\begin{array}{c}137(28.4) \\
{[24.2,33.0]}\end{array}$ & $\begin{array}{c}122(26.5) \\
{[21.8,31.9]}\end{array}$ & $469(100.0)$ \\
\hline $45-49$ & $118(25.6)$ & $49(10.9)$ & $131(31.5)$ & $137(31.9)$ & $435(100.0)$ \\
\hline
\end{tabular}




\begin{tabular}{|c|c|c|c|c|c|}
\hline \multicolumn{6}{|c|}{ Table 4. Ctd } \\
\hline \multirow[t]{2}{*}{$50-54$} & $113(20.5)$ & $57(10.4)$ & $168(32.2)$ & $211(36.8)$ & $549(100.0)$ \\
\hline & {$[16.6,25.1]$} & {$[7.02,15.1]$} & {$[24.7,40.8]$} & {$[29.5,44.9]$} & \\
\hline \multirow[t]{2}{*}{$55-59$} & $110(16.6)$ & $58(8.8)$ & $181(29.5)$ & $284(45.1)$ & $633(100.0)$ \\
\hline & {$[13.3,20.5]$} & {$[6.25,12.2]$} & {$[25.7,33.6]$} & {$[39.7,50.6]$} & \\
\hline \multirow[t]{2}{*}{$60-64$} & $91(16.2)$ & $53(9.5)$ & $159(28.5)$ & $272(45.9)$ & $575(100.0)$ \\
\hline & {$[12.5,20.7]$} & {$[6.73,13.1]$} & {$[23.7,33.8]$} & {$[41.6,50.2]$} & \\
\hline \multirow[t]{2}{*}{$65-69$} & $64(13.3)$ & $45(9.4)$ & $131(26.6)$ & $257(50.7)$ & 497 (100.0) \\
\hline & {$[9.76,17.9]$} & {$[6.71,13.0]$} & {$[21.1,33.0]$} & {$[44.3,57.0]$} & \\
\hline \multirow[t]{2}{*}{ Total } & $2137(46.1)$ & $554(9.6)$ & $1576(26.3)$ & $1535(18.0)$ & $5802(100.0)$ \\
\hline & {$[42.8,49.5]$} & {$[7.86,11.7]$} & {$[24.5,28.2]$} & {$[16.1,20.1]$} & \\
\hline
\end{tabular}

group, which was slightly lower. The comparison between the prevalence of stage 1 and 2 hypertension across the age groups in both sexes showed a higher prevalence in men (Tables 3 and 4).

The prevalence of elevated BP, stage 1 and 2 hypertension, for men and women is presented in Figure 1. The prevalence of elevated BP in men has decreased from 20 years $(20.5 \%)$ to 65 years $(8.8 \%)$. However, in women, the prevalence of elevated BP remained roughly constant
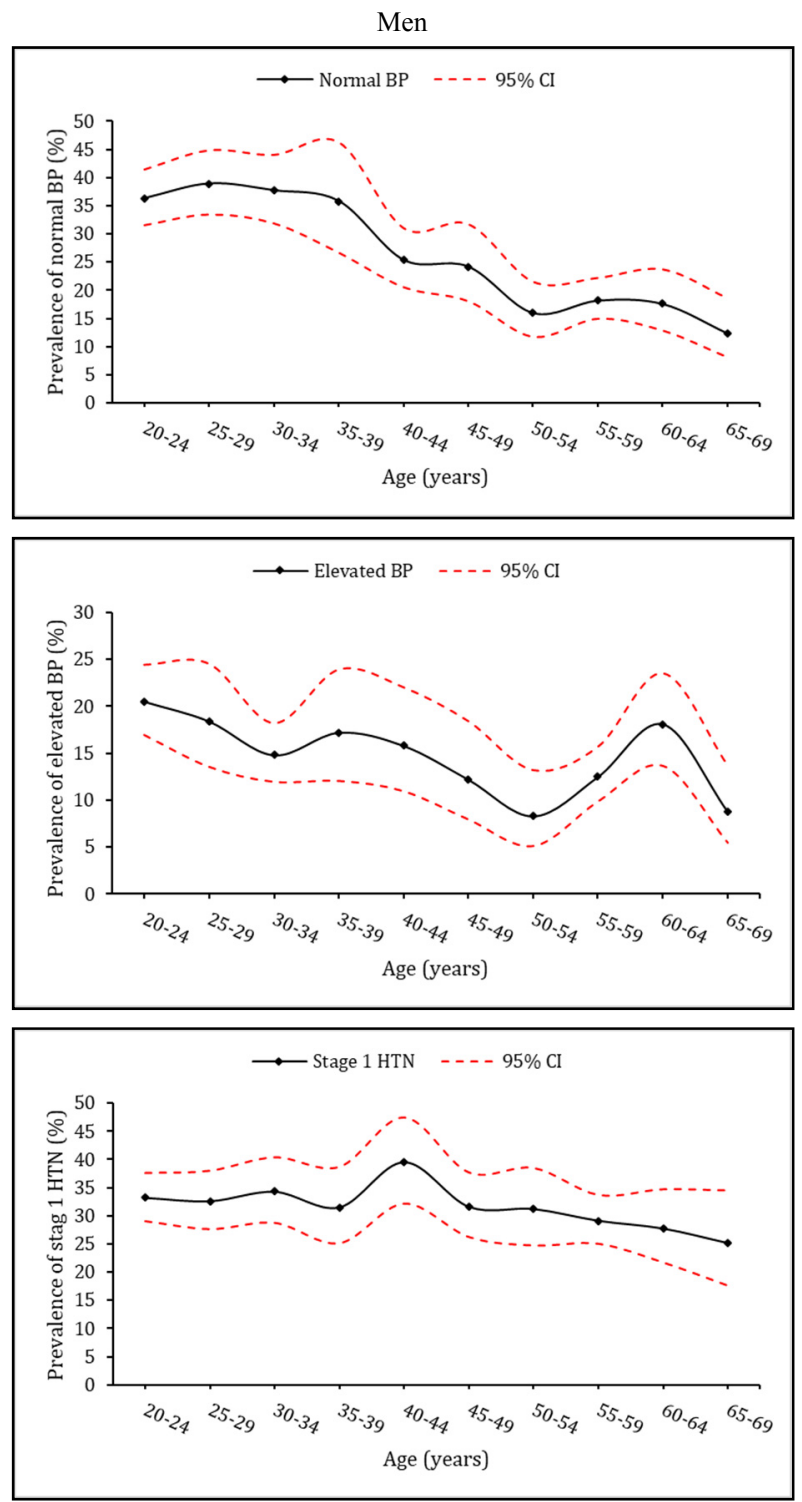

in 20-69 age group. The prevalence of stage 1 hypertension in men and women almost follows the same pattern. This prevalence remains roughly constant in both sexes aged 20-69 years. Only an increase in the prevalence of stage 1 hypertension occurs in men aged 40-44 years.

Prevalence of stage 2 hypertension was different in men and women. The prevalence of stage 2 hypertension was $10.1 \%$ in men aged 20 to 24 years, which increased to $44.5 \%$ in $50-54$ age groups. However, this prevalence
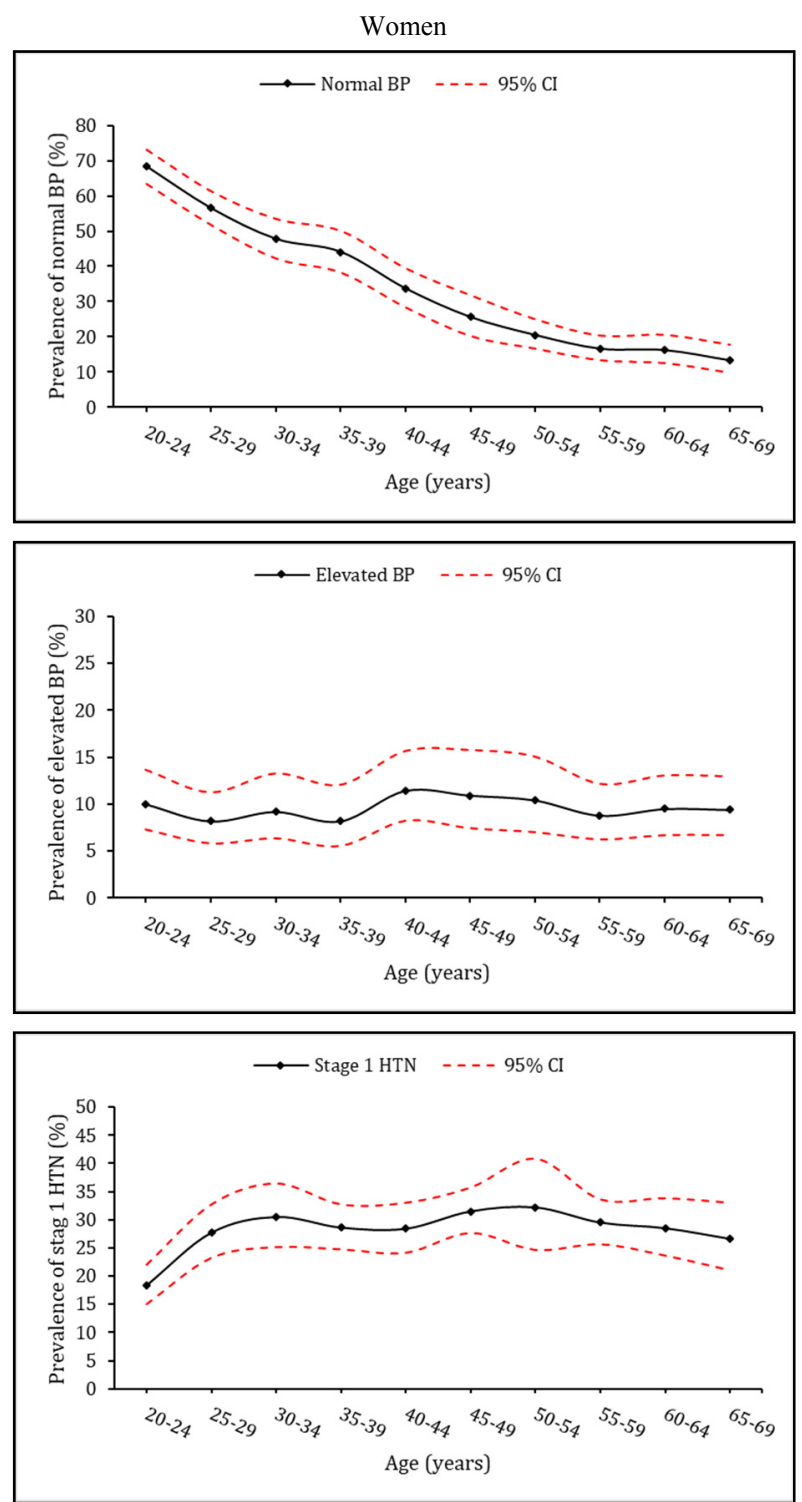

Fig. 1. Prevalence of normal blood pressure (BP), elevated BP and stage 1 and 2 hypertension (HTN) with $95 \%$ confidence interval (95\% CI) according to age group and sex 

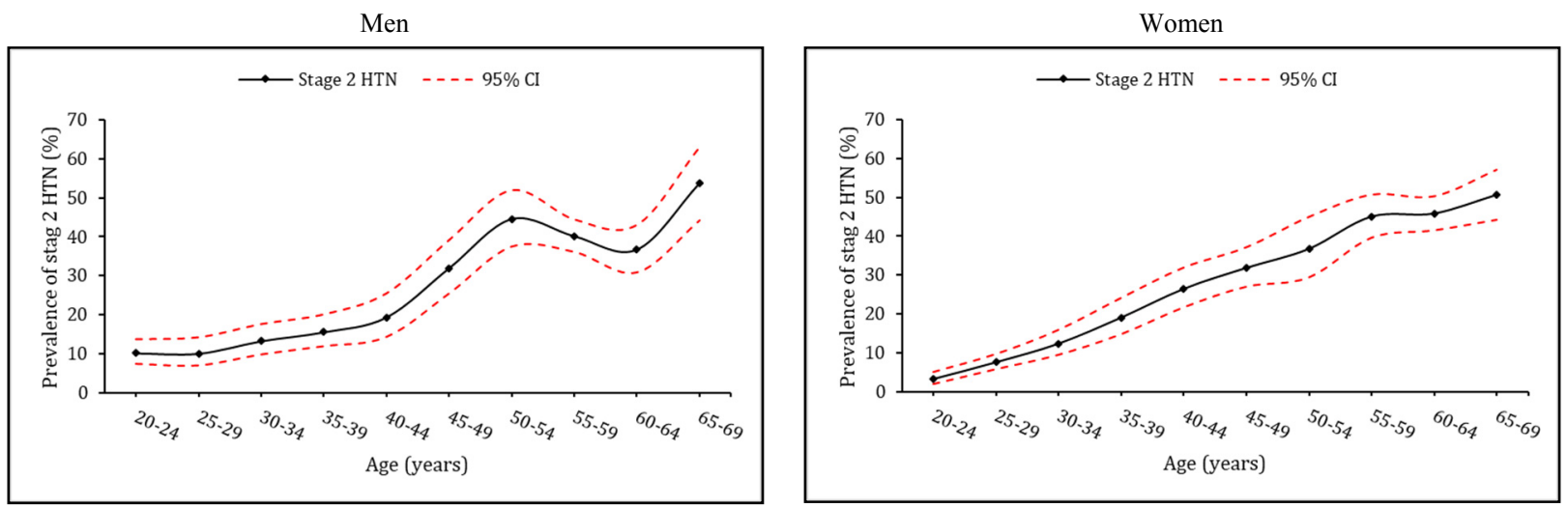

Fig. 1. Ctd

Men

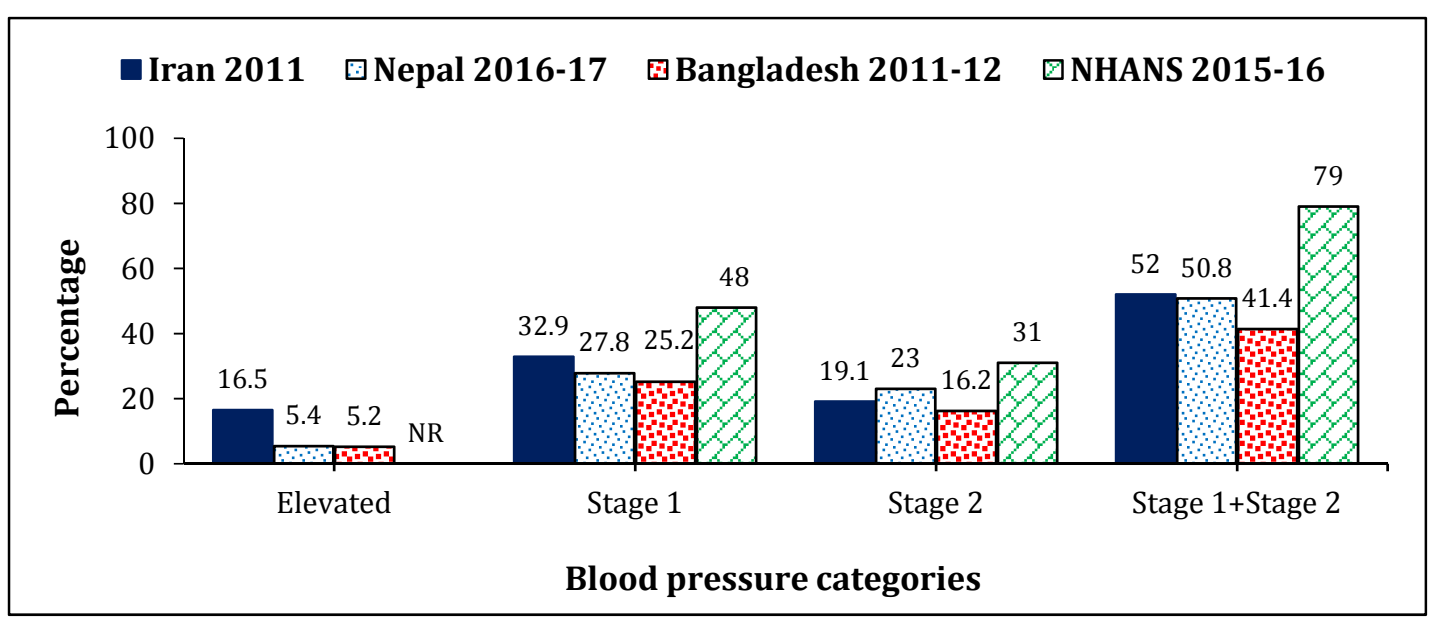

Women

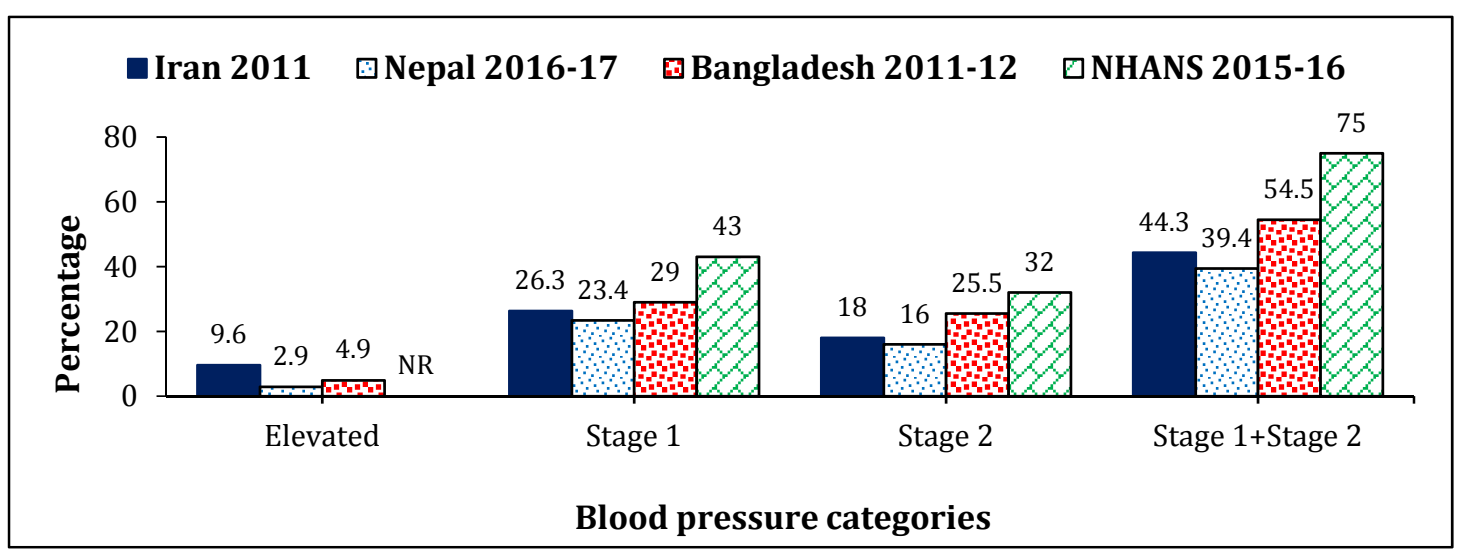

Fig. 2. The prevalence of hypertension in Iranian, American, Bangladeshi, and Nepali adults based on sex using the new ACC/AHA guidelines. NR: Not reported.

decreased to $40.1 \%$ and $36.7 \%$ in $55-59$ and $60-64$ age groups, respectively. Then, it increased to $53.7 \%$ in those aged 65-69 years. Unlike men, in women, the prevalence of stage 2 hypertension had a steady increase in women. The prevalence of stage 2 hypertension was $3.3 \%$ in women aged $20-24$ years, which raised to $45.1 \%$ in $55-59$ age group and $50.7 \%$ in $65-69$ age group.

\section{Discussion}

For the first time, the findings of this study, based on ACC/AHA guidelines, showed that the prevalence of hypertension in men and women is higher than previous estimates in Iran. Overall prevalence of hypertension in Iranian men and women was $52.0 \%$ and $44.3 \%$, respectively. By contrast, according to the JNC-7 guidelines, the preva- 
lence of hypertension in men and women was $25.8 \%$ and $27.6 \%$, respectively (2). In addition, about $16.5 \%$ of men and $9.6 \%$ of women were categorized as having elevated blood pressure who are prone to hypertension. The prevalence of hypertension in men and women follows a different pattern according to age. The prevalence of hypertension in women up to the age of 55 years was lower than men, while in ages older than 55 , the prevalence was similar in both sexes.

The ACC/AHA guideline presented in 2017 caused considerable changes in the report of prevalence of hypertension in communities. In the United States, the prevalence of stage 1 and 2 hypertension was $48 \%$ and $31 \%$ in men and $43 \%$ and $32 \%$ in women, respectively (5). According to the findings of this study, the prevalence of hypertension in the United States is far higher than the Iranian population. The prevalence of stage 1 and stage 2 hypertension in Iranian men and women was $32.9 \%$, $19.1 \%$, and $26.3 \%$ and $18 \%$, respectively. These values were similar to those in other Asian countries such as Nepal (12) and Bangladesh (13), with the prevalence of stage 1 hypertension in men varying from $25.2 \%$ and $27.8 \%$ and in women from $23.4 \%$ to $29 \%$, respectively. This range for stage 2 hypertension was $16.2 \%$ to $23 \%$ and $25.5 \%$ to $16 \%$, respectively (Fig. 2).

Identifying at-risk individuals is highly important in primary prevention. Therefore, the AHA / ACC 2017 guideline defined a more sensitive cutoff for elevated blood pressure than JNC-7 report. In Iran, 16.5\% of men and $9.6 \%$ of women have been classified as having elevated blood pressure, which is higher than Nepal $(5.4 \%$ in men and $2.9 \%$ in women) and Bangladesh (5.2\% in men and $4.9 \%$ in women) (Fig. 2). It seems that the prevalence of hypertension in Iran is higher than low-income countries and lower than high-income countries.

The reason for the difference in the prevalence of hypertension in men and women by age can be attributed to their physiological differences. At premenopausal ages (50 years or less), estrogen secretion causes women to have lower blood pressure than men. However, at menopause, the decrease in the level of estrogen reduces its protective effect on blood pressure (14).

Zero or 5 end-digit preference is one of the most common errors in most large-scale surveys (15). The prevalence of this error is very high in the assessment of BP. For example, in a study on 16433 individuals in China, the prevalence of zero end-digit preference was found to be $52.5 \%$ and $63.5 \%$ for SBP and DBP, respectively (16). These values were $64 \%$ and $62 \%$, respectively, in another study in New Zealand (17). However, in SuRFNCD 2011 in Iran, which was used in this study, zero end-digit preference was only $16.1 \%$. Using a national data from Iran is one of the strengths of this research. In addition, this study was one of the few reports in a developing country which used ACC/AHA 2017 guideline to assess hypertension. Also, in the analysis of the present data in estimation of new BP categories in adults, the weighted analysis of the data was undertaken because of sampling scheme. This strategy provided a more accurate estimate of the prevalence of hypertension.
One of the limitations of the present study was the use of data collected in 2011, as the blood pressure status in Iran may have changed since then. However, in a previous study, it was found that the prevalence of hypertension in Iran over 1999, 2003, 2007, and 2011 did not change significantly (2). Therefore, one might assume that even the amount of change from 2011 up to now (2018), is negligible and the results of this study can be used by health authorities in the country.

\section{Conclusion}

This study revealed that adopting the 2017 ACC/AHA guidelines provided a new prevalence of adult hypertension $(48.2 \%)$ in Iran. In this study, men were more hypertensive compared to women. In addition, the prevalence was higher in older adults irrespective of sex. This new prevalence is higher compared to that of previous studies which were done based on the JNC-7 guidelines. To decrease this alarming increase, urgent measures, such as effective health policies, healthy and positive change in lifestyle, proper diet, regular exercise, sensitization and awareness programs, and regular medical check-ups are highly recommended.

\section{Acknowledgment}

This research has been supported by Tehran University of Medical Sciences \& health Services grant (Grant number: 97-02-27-38625).

\section{Conflict of Interests}

The authors declare that they have no competing interests.

\section{References}

1. Hosseini M, Baikpour M, Yousefifard M, Fayaz M, Koohpayehzadeh J, Ghelichkhani P, et al. Blood pressure percentiles by age and body mass index for adults. EXCLI J. 2015;14:465.

2. Hosseini M, Yousefifard M, Baikpour M, Rafei A, Fayaz M, Heshmat $\mathrm{R}$, et al. Twenty-year dynamics of hypertension in Iranian adults: age, period, and cohort analysis. J Am Soc Hypertens. 2015;9(12):925-34.

3. Hosseini M, Ataei N, Aghamohammadi A, Yousefifard M, Taslimi S, Ataei F. The relation of body mass index and blood pressure in Iranian children and adolescents aged 7-18 years old. Iran J Public Health. 2010;39(4):126.

4. Ibrahim MM, Damasceno A. Hypertension in developing countries. Lancet 2012;380(9841):611-9.

5. Whelton PK, Carey RM, Aronow WS, Casey DE, Collins KJ, Himmelfarb CD, et al. 2017 ACC/AHA/AAPA/ABC/ACPM/AGS/ $\mathrm{APhA} / \mathrm{ASH} / \mathrm{ASPC} \mathrm{NMA} / \mathrm{PCNA}$ guideline for the prevention, detection, evaluation, and management of high blood pressure in adults: a report of the American College of Cardiology/American Heart Association Task Force on Clinical Practice Guidelines. J Am Coll Cardiol. 2018;71(19):e127-e248.

6. Rao MV, Qiu Y, Wang C, Bakris G. Hypertension and CKD: Kidney Early Evaluation Program (KEEP) and National Health and Nutrition Examination Survey (NHANES), 1999-2004. Am J Kidney Dis. 2008;51(4):S30-S7.

7. Muntner P, Carey RM, Gidding S, Jones DW, Taler SJ, Wright JT, Jr., et al. Potential US Population Impact of the 2017 ACC/AHA High Blood Pressure Guideline. Circulation. 2018;137(2):109-18.

8. Khera R, Lu Y, Lu J, Saxena A, Nasir K, Jiang L, et al. Impact of 2017 ACC/AHA guidelines on prevalence of hypertension and eligibility for antihypertensive treatment in United States and China: nationally representative cross sectional study. BMJ (Clinical research ed). 2018;362:k2357

9. Haghdoost AA, Sadeghirad B, Rezazadehkermani M. Epidemiology 
and heterogeneity of hypertension in Iran: a systematic review. Arch Iran Med. 2008;11(4):444-52.

10. Mohammadi M, Mirzaei M. Population-attributable fraction of hypertension associated with obesity, abdominal obesity, and the joint effect of both in the Central Provinces of Iran. JJ Epidemiol Glob Health. 2017;7(1):71-9.

11. Koohpayehzadeh J, Etemad K, Abbasi M, Meysamie A, Sheikhbahaei S, Asgari F, et al. Gender-specific changes in physical activity pattern in Iran: national surveillance of risk factors of noncommunicable diseases (2007-2011). Int J Public Health. 2014;59(2):231-41

12. Al Kibria GM, Swasey K, Angela K, Mirbolouk M, Sakib MN, Sharmeen A, et al. Estimated change in prevalence of hypertension in Nepal following application of the 2017 ACC/AHA guideline. JAMA Network Open. 2018;1(3):e180606-e.

13. Al Kibria GM, Swasey K, Choudhury A, Burrowes V, Stafford KA, Uddin SI, et al. The new 2017 ACC/AHA guideline for classification of hypertension: changes in prevalence of hypertension among adults in Bangladesh. J Hum Hypertens. 2018:1.

14. Hinton Jr AO, He Y, Xia Y, Xu P, Yang Y, Saito K, et al. Estrogen receptor- $\alpha$ in the medial amygdala prevents stress-induced elevations in blood pressure in females. Hypertension. 2016;67(6):1321-30.

15. Yaseri M, Afarideh M, Hosseini M, Yousefifard M, Rafei A, Koohpayehzadeh J, et al. Zero and Five End-Digit Preference and Blood Pressure Quality of Care Revisited. Arch Iran Med. 2017;20(10):633-9.

16. Wang Y, Wang Y, Qain Y, Zhang J, Tang X, Sun J, et al. Longitudinal change in end-digit preference in blood pressure recordings of patients with hypertension in primary care clinics: Minhang study. Blood Press Monit. 2015;20(2):74-8.

17. Broad J, Wells S, Marshall R, Jackson R. Zero end-digit preference in recorded blood pressure and its impact on classification of patients for pharmacologic management in primary care - PREDICT-CVD-6. Br J Gen Pract. 2007;57(544):897-903. 